\title{
Molecular $\mathrm{CsF}_{5}$ and $\mathrm{CsF}_{2}^{+* *}$
}

\author{
Andrey Yu. Rogachev, Mao-sheng Miao, Gabriel Merino, and Roald Hoffmann*
}

\begin{abstract}
D_{5 h}$ star-like $C s F_{5}$, formally isoelectronic with known $\mathrm{XeF}_{5}{ }^{-}$ion, is computed to be a local minimum on the potential energy surface of $\mathrm{Cs}_{5}$, surrounded by reasonably large activation energies for its exothermic decomposition to $C s F+$ $2 F_{2}$, or to $\mathrm{Cs}_{3}$ (three isomeric forms) $+F_{2}$, or for rearrangement to a significantly more stable isomer, a classical $\mathrm{Cs}^{+}$ complex of $\mathrm{F}_{5}{ }^{-}$. Similarly the $\mathrm{CsF}_{2}{ }^{+}$ion is computed to be metastable in two isomeric forms. In the more symmetrical structures of these molecules there is definite involvement in bonding of the formally core $5 p$ levels of Cs.
\end{abstract}

$N_{c}$ valence and core orbitas become good reasons, as the energetic separation of all potentially occupiable levels becomes small. For instance, it has been suspected that some sub-valence levels of uranium are involved in uranium-ligand bonding. ${ }^{[1]}$ And a compound of mercury in oxidation state $\mathrm{IV}, \mathrm{HgF}_{4}$, involving the d block in bonding, was predicted and observed. ${ }^{[2,3]}$ This is at $p=1 \mathrm{~atm}$; at higher pressures, calculated compositions of matter stepping well outside of normal valence regularities abound. ${ }^{[4]}$

We report herein, at least in theory, another clear $p=$ $1 \mathrm{~atm}$ instance of the involvement of orbitals thought to be core orbitals in bonding. Our investigation derives from the theoretical finding of $\mathrm{CsF}_{5}$ and $\mathrm{CsF}_{2}^{+}$molecular units in a study of cesium fluorides under elevated pressure. ${ }^{[5]}$ Given that the molecules are isoelectronic with known species $\mathrm{XeF}_{5}{ }^{-[6]}$ and $\mathrm{XeF}_{2}$, respectively, we decided to examine their potential (even if fleeting) existence as discrete molecules at ambient or low pressure. ${ }^{[7]}$ We predict the thermodynamic metastability and likely kinetic persistence of molecular $\mathrm{CsF}_{5}$ and $\mathrm{CsF}_{2}{ }^{+}$. In certain isomers of these compounds, $\mathrm{Cs}$ is in formal oxidation state $\mathrm{V}$ and III, respectively, and forms polar $\mathrm{Cs}-\mathrm{F}$ bonds. ${ }^{[8]}$ We note some excellent recent experimental and theoretical work on $\mathrm{CsF}_{3}$ and $\mathrm{CsF}_{5}$ ion pairs by VentSchmidt et al. ${ }^{[9]}$

To obtain reliable results, a reasonably high level of theory has to be used and relativistic effects need to be taken into account (details in the Methods Section at the end of the paper, and in the Supporting Information). Computational pathologies pose obstacles, as we will describe.

The $D_{5 h} \mathrm{CsF}_{5}$ structure is a local minimum (Hessian matrix eigenvalues all positive) in our calculations (PBE0/ TZVP/ZORA level; the same geometry is obtained with other methods; please see the Methods Section and the Supporting Information), and is shown in Figure 1. The star-

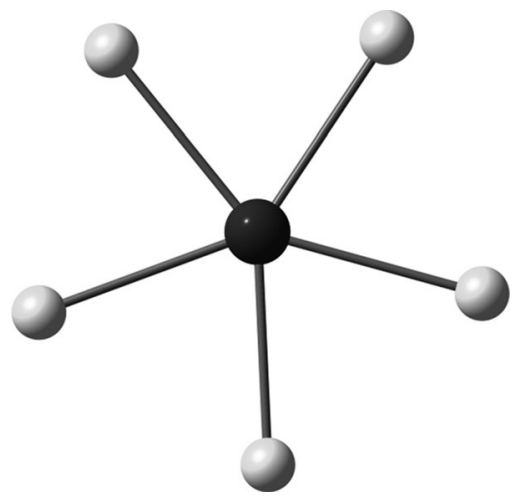

Figure 1. Calculated equilibrium geometry of $\mathrm{CsF}_{5}$.

like planar geometry mirrors its isoelectronic anionic $\mathrm{XeF}_{5}{ }^{-}$. The calculated $\mathrm{Cs}-\mathrm{F}$ distance is $2.05 \AA^{\left[{ }^{[10]}\right.}$ Essentially the same results were obtained by the $\operatorname{CCSD}(\mathrm{T})$ method $(\mathrm{Cs}-\mathrm{F}$ distance is $2.08 \AA$, see the Supporting Information for details), thus providing important support for the reliability of PBE0 geometry calculations.
[*] Dr. A. Yu. Rogachev

Dept. of Biological and Chemical Sciences, Illinois Institute of Technology (USA)

Dr. M.-S Miao

Department of Chemistry and Biochemistry, California State

University Northridge (USA)

and

Beijing Computational Science Research Center (P.R. China)

Dr. G. Merino

Departamento de Física Aplicada, Centro de Investigación y de Estudios Avanzados, Unidad Mérida (México)

Dr. R. Hoffmann

Department of Chemistry and Chemical Biology

Cornell University, Baker Laboratory

Ithaca NY 14853-1301 (USA)

E-mail:rh34@cornell.edu
[***] We are grateful to the National Science Foundation for its support of this work through Research Grant CHE-0910623 to Cornell University. A.Yu.R also greatly acknowledges support from the Illinois Institute of Technology (IIT) through startup funding. M.S.M. thanks for support through the MRSEC program (NSFDMR1 121053) and the ConvEne-IGERT Program (NSF-DGE 0801627). The Moshinsky Foundation supports the work in Mérida. Our calculations were carried out at the computational facilities of KAUST (King Abdullah University of Science and Technology) Supercomputing Laboratory and IIT. Some earlier test calculations were performed on NSF-funded XSEDE resources (TGDMR130005). We thank L. Andrews, S. Riedel, and a reviewer for their criticism of the original version of this work, and $\mathrm{H}$. Rzepa for discussion.

$\square$ Supporting information for this article is available on the WWW under http://dx.doi.org/10.1002/anie.201500402. 
How stable (or unstable) is $D_{5 h} \mathrm{CsF}_{5}$ with respect to its expected decomposition products $\mathrm{CsF}+2 \mathrm{~F}_{2}$ ? That turned out to be a difficult question to answerthere was a huge discrepancy of more than $60 \mathrm{kcal}$ $\mathrm{mol}^{-1}$ in the $\Delta E$ between $\operatorname{CCSD}(\mathrm{T}) / \mathrm{TZVP}$ (or CCSD(T)/QZVPP or MP2/TZVP, all with ZORA corrections) calculations on one hand, and PBE0 calculations with the same basis sets on the other. The PBE0/TZVP/ZORA level (the results are also
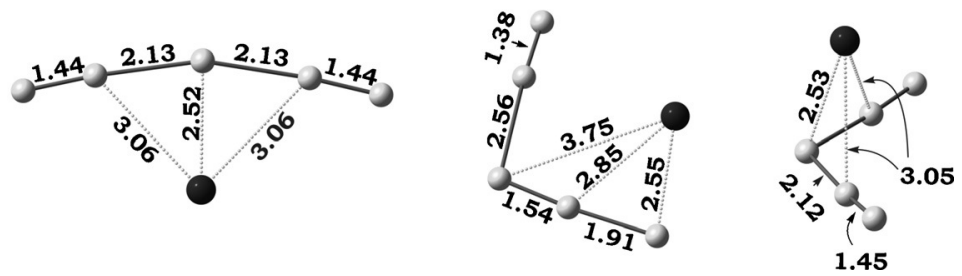

Figure 3. Three calculated less-symmetrical low-energy structures for $\mathrm{CsF}_{5}$. supported by other methods ${ }^{[11]}$ ) gives $-45 \mathrm{kcal} \mathrm{mol}^{-1}$ as the energy for decomposition. The products have computed distances of $2.375 \AA$ for CsF (experimental: $2.345 \AA^{[12]}$ ) and $1.383 \AA$ for $F_{2}$ (experimental: $1.412^{[13]}$ ). To get more reliable estimations, we then turned to multireference (MR) perturbation theory in the XMCQDPT2 variant (these results will be used throughout the subsequent discussion; please see the Methods Section and the Supporting Information for details). This method gives $\Delta E=-44 \mathrm{kcal} \mathrm{mol}^{-1}$, which agrees well with the DFT value. Subsequent analysis of the wavefunction revealed that the leading closed-shell singlet configuration contributes $83 \%$ (see the Supporting Information for details). Importantly, the weight of any other electronic configuration that contributes to the final wavefunction was found to be no more than $2 \%$. We conclude that the ground state of $D_{5 h} \mathrm{CsF}_{5}$ can be approximately described by single-reference methods.

The decomposition of $\mathrm{CsF}_{5}$ to $\mathrm{CsF}+2 \mathrm{~F}_{2}$ is allowed (no level crossings) along a least-motion reaction coordinate. The transition state (TS) we found for this process is shown in Figure 2 ; the same results were obtained with $\left(C_{2 v}\right)$ and

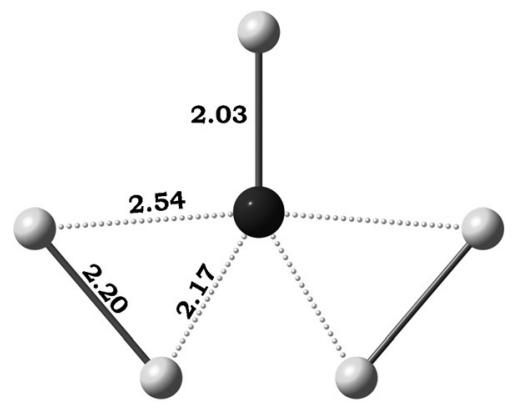

Figure 2. Transition-state geometry for decomposition of $\mathrm{CsF}_{5}$ to $\mathrm{CsF}$ and two molecules of $F_{2}$. Selected geometrical parameters are shown.

without $\left(C_{1}\right)$ symmetry restrictions applied. An activation energy of $+39 \mathrm{kcal} \mathrm{mol}^{-1}$ was computed for the process at the XMCQDPT2 level. In contrast to the ground state of the starlike isomer, the $C_{2 v}$ TS wavefunction is substantially multireference; it corresponds to an open-shell singlet configuration, with two leading configurations of equal weight (44\%). Still another highly exothermic channel is to $\mathrm{F}_{2}$ and $\mathrm{CsF}_{3}$ (the $\mathrm{Cs}^{+}$complex of the trifluoride ion, ${ }^{[8]}$ more on this species soon $\left.^{[14]}\right)$. This channel could be viewed as an asymmetric fragmentation, with a computed $\Delta E$ of $-9 \mathrm{kcal} \mathrm{mol}^{-1}$ and $E_{\mathrm{a}}$ of $+73 \mathrm{kcal} \mathrm{mol}^{-1}$. The TS was found to be open-shell singlet in nature, $70 \%$ coming from two leading configurations.

Symmetry is not a good guide to stability; in addition to the beautiful star-shaped structure for $\mathrm{CsF}_{5}$ (and weakly bound complexes of $\mathrm{CsF}$ and two $\mathrm{F}_{2}$ molecules described in the Supporting Information), we have also discovered some less-symmetrical and quite stable $\mathrm{CsF}_{5}$ structures. Three local minima are shown in Figure 3; they may be viewed as $\mathrm{Cs}^{+}$ complexes of $\mathrm{F}_{5}^{-[9,15]}$ or $\mathrm{F}_{3}^{-[15 \mathrm{c}, 16]}$ and $\mathrm{F}_{2}$, stabilized much as Ault and Andrews originally reasoned for $\mathrm{CsF}_{3} \cdot{ }^{\left[{ }^{8]}\right.}$ The structures are 42 to $49 \mathrm{kcal} \mathrm{mol}^{-1}$ more stable than the $D_{5 h}$ star. ${ }^{[17]}$ All these isomers of $\mathrm{CsF}_{5}$ can be approximately described by a closed-shell singlet electronic configuration with a weight of the leading term of more than $86 \%$. The barriers between the three $\mathrm{Cs}^{+}+\mathrm{F}_{5}^{-}$structures were found to be small (no more than $3 \mathrm{kcalmol}^{-1}$, see the Supporting Information); it appears this is a single easily deformed family of structures. In terms of geometrical distortions, we think these structures are far away from the star, and a distortion to them will encounter substantial barriers. The various $\mathrm{CsF}_{5}$ isomers have quite distinct vibrations (see the Supporting Information, which also has a calibration on $\mathrm{F}_{2}$ and $\mathrm{F}_{3}{ }^{-}$), so they should be distinguishable.

We note that an extended $\mathrm{CsF}_{5}$ phase containing $\mathrm{Cs}^{+}$and $\mathrm{F}_{5}{ }^{-}$ions, in a relative geometry resembling the right-hand structure in Figure 3, has been calculated to be stable at $p=$ $1 \mathrm{~atm}$ by Zhu, Oganov, and Zeng. ${ }^{[18]}$

Like the $\mathrm{XeF}_{5}{ }^{-}$ion, $\mathrm{CsF}_{5}$ has a well-defined high-lying molecular orbital (MO) that is mainly $5 \mathrm{p}_{z}$ on Cs (the two MOs are illustrated in the Supporting Information). For $\mathrm{CsF}_{5}$, this MO lies $0.69 \mathrm{eV}$ lower than the HOMO of the molecule. We have recently predicted relatively high stability for organometallic Lewis acid adducts of $\mathrm{XeF}_{5}{ }^{-}{ }^{[19]}$ And we find that a hypothetical transition-metal complex $\left[\mathrm{W}(\mathrm{CO})_{5} \mathrm{CsF}_{5}\right], \mathrm{W}-$ Cs bonded, corresponds to a local minimum. Weakly bound as it is, by $6 \mathrm{kcal} \mathrm{mol}^{-1}$ (at the PBE0 level, see the SI), this adduct represents a rare case of activated reactivity of near-core (in this case $5 p_{z}$ ) orbitals.

We turn next to the $\mathrm{CsF}_{2}{ }^{+}$ion which is isoelectronic with $\mathrm{XeF}_{2}$. Interestingly, two local minima emerge on its potential surface as shown in Figure 4. The bent isomer is unstable by a)

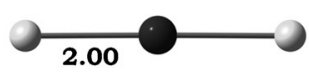

b)

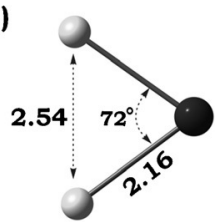

Figure 4. Equilibrium geometry configurations for a) linear and b) bent isomers of $\mathrm{CsF}_{2}^{+}$. 
about $25 \mathrm{kcal} \mathrm{mol}^{-1}$ relative to the linear one, as calculated at the XMCQDPT2 level. For both isomers, the ground state is a singlet. However, for the linear form, the contribution from the leading (closed-shell) configuration is $86 \%$, while another $13 \%$ come from a second component. At the same time, for the bent analogue, the two leading configurations have weights of $57 \%$ and $41 \%$ and correspond to closed-shell and open-shell singlets, respectively. Both systems thus appear as significantly multireference in nature.

The reaction $\mathrm{CsF}_{2}^{+} \rightarrow \mathrm{Cs}^{+}+\mathrm{F}_{2}$ is calculated to be exothermic with $\Delta E=-61 \mathrm{kcal} \mathrm{mol}^{-1}$ (again in excellent agreement with the DFT value of $-59 \mathrm{kcal} \mathrm{mol}^{-1}$ ). Calculations of the reaction pathway led to a two-step mechanism (Figure 5) with

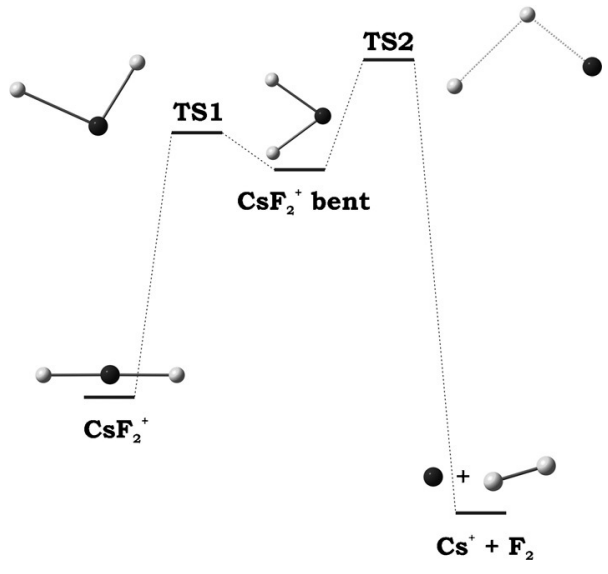

Figure 5. Schematic energy profile for the reaction $\mathrm{CsF}_{2}{ }^{+} \rightarrow \mathrm{Cs}^{+}+\mathrm{F}_{2}$

one intermediate, the abovementioned bent isomer of the $\mathrm{CsF}_{2}^{+}$ion. Despite the large exothermicity, this reaction encounters a high reaction barrier $\left(E_{\mathrm{a}}(1)=+29 \mathrm{kcal} \mathrm{mol}^{-1}\right.$, the first barrier of the reaction considered). The asymmetrical $C_{\mathrm{s}}$ transition states are drawn out in the Supporting Information. As we have seen already in the case of $\mathrm{CsF}_{5}$ systems, both transition states, TS1 and TS2, are highly multireference (see the Supporting Information).

We mentioned above an asymmetric $\mathrm{CsF}_{3}$ species, a complex of $\mathrm{Cs}^{+}$with $\mathrm{F}_{3}^{-}$. Actually, we located at least three local minima for this stoichiometry. The low-symmetry structure of the most stable isomer is close to the geometry of the righthand structure in Figure 3, with $\mathrm{F}_{2}$ removed (see the Supporting Information). A second structure derives from a $D_{3 h}$ star-shaped molecule. Such a species would share the electronic characteristics of the isoelectronic (and known $\left.{ }^{[20,21]}\right) \mathrm{XeF}_{3}{ }^{-}$ion in being a classic Jahn-Teller system. Two electrons would occupy an e' orbital, with deformations to $\mathrm{T}$ and $\mathrm{Y}$ shapes expected. ${ }^{[20]}$ The $\mathrm{T}$ shape for both $\mathrm{XeF}_{3}{ }^{-}$ and $\mathrm{CsF}_{3}$ is not a local minimum, while the $\mathrm{Y}$ shape is. ${ }^{[20-23]}$ The energy of this minimum is about $32 \mathrm{kcal} \mathrm{mol}^{-1}(26 \mathrm{kcal}$ $\mathrm{mol}^{-1}$ by DFT) above the asymmetric structure mentioned above. Finally, there is a pyramidal $C_{3 v}$ isomer, $46 \mathrm{kcal} \mathrm{mol}^{-1}$ ( $38 \mathrm{kcal} \mathrm{mol}^{-1}$ at the PBE0 level) higher in energy. The T and Y shaped isomers are closed-shell singlets, with $83 \%$ and $90 \%$ contribution, respectively, from the leading configura- tion. In contrast, the pyramidal $\mathrm{CsF}_{3}$ has an open-shell singlet as the ground state.

What about the bonding in these cesium fluorides? The question refers to the structures that are not ion pairs of $\mathrm{Cs}^{+}$ with $\mathrm{F}_{3}{ }^{-}$or $\mathrm{F}_{5}{ }^{-}$. Oxidation states are a convenient fiction, so one must not conclude from them that one breaks into the hypothetical "core" $5 \mathrm{~s}$ and $5 \mathrm{p}$ orbitals of Cs. But a population analysis in fact indicates that $5 \mathrm{p}$ orbitals are involved in the bonding: the total population of 5 p orbitals in $\mathrm{CsF}_{5}(q(\mathrm{Cs})=+$ 2.40) and $\mathrm{CsF}_{2}^{+}(q(\mathrm{Cs})=+1.79)$ is $4.41 \mathrm{e}$ and $5.12 \mathrm{e}$, respectively. In the case of $\mathrm{CsF}_{5}, \mathrm{Cs} 5 \mathrm{p}_{x}$ and $5 \mathrm{p}_{y}$ orbitals both are occupied by about 1.20 e (notably below 2.00 e, owing to the participation of $5 \mathrm{p}_{x}$ and $5 \mathrm{p}_{y}$ in hypervalent bonding), whereas the population of $5 \mathrm{p}_{z}$ (which does not take part in bonding) is exactly 2.00 e. A similar situation occurs for the $\mathrm{CsF}_{2}{ }^{+}$ion, in which only the $5 \mathrm{p}_{x}$ orbital on Cs has a low occupancy of $1.25 \mathrm{e}$ through its participation in $4 \mathrm{e}-3 \mathrm{c}$ bonding, whereas two other orbitals, $5 \mathrm{p}_{y}$ and $5 \mathrm{p}_{z}$, are close to doubly occupied (1.88 e and $2.00 \mathrm{e}$, respectively). In contrast, the same analysis performed for $\mathrm{CsF}(q(\mathrm{Cs})=+0.93)$ revealed that none of the $5 \mathrm{p}$ orbitals of the cesium center (5p total population is essentially $6.00 \mathrm{e}$ ) is involved in interaction with fluorine atoms.

We look forward to the detection of these intriguing higher fluorides of cesium.

\section{Methods}

DFT geometry explorations were performed with the help of the PBE0 correlation-exchange functional (within the RIJCOSX approximation). Full-electron relativistically recontracted basis sets of triple$\mathrm{Z}$ quality (TZVP) were used for all atoms. Scalar relativistic effects were incorporated by applying the zero-order regular approximation (ZORA); the influence of spin-orbit coupling (SO) was evaluated through the ZORA approximation at the PBE0/TZ2P level with help of the ADF program. ${ }^{[24]}$ The methods used were thus PBE0/QZVPP/ ZORA; PBE0/TZVP/DKH2; MP2/TZVP/ZORA. The SO contribution was found to be about $1.33 \mathrm{kcal} \mathrm{mol}^{-1}$ and, thus, considered as negligible. ${ }^{[25]}$

Test calculations were also carried out for PBE0/TZVP-optimized geometries at the following levels of theory: MP2/TZVP and its orbital-optimized spin-scaled version OO-SCS-MP2/TZVP, $\operatorname{CCSD}(\mathrm{T}) / \mathrm{TZVP}$ and CCSD(T)/QZVPP (all with help of the RIJCOSX approximation). These calculations were performed by using the ORCA program suite (version 2.9.1). ${ }^{[6]}$ The natural bond orbitals (NBOs, GENNBO (version 5.0) program) of the optimized geometries were then examined to gain some insight into the electronic structures. The nature of all stationary points on the potential energy surfaces (PES) was determined by calculation of the full Hessian matrix and the harmonic vibrational frequencies. All structures reported were found to be true minima on corresponding PES (no imaginary frequencies), whereas all TSs were found to possess only one imaginary frequency, corresponding to the transition between reactants and the products intended. The nature of all TSs was probed through the IRC technique connecting the target products and reactants. IRC calculations were carried out with help of the Firefly program (version 8.0) at the PBE0/def2-TZVP level of theory.

All MR calculations were performed for PBE0-optimized geometries. The active space includes the full valence space for fluorine atoms ( 7 electrons distributed over 1 orbital of s-type and 3 orbitals of p-type; altogether 7 electrons and 4 orbitals per $\mathrm{F}$ atom). For the cesium atom, 1 electron in the $6 \mathrm{~s}$ orbital, 8 electrons in the $5 \mathrm{~s}$ and $5 \mathrm{p}$ orbitals were included in the active space. This approach produced 
(44 e, 22 orb), (30 e, 16 orb), and (22 e,12 orb) active spaces for $\mathrm{CsF}_{5}$, $\mathrm{CsF}_{3}$, and $\mathrm{CsF}_{2}^{+}$, respectively. Converged CASSCF wavefunctions were then used as reference wavefunctions for subsequent calculations by the XMCQDPT2 method. ${ }^{[27]}$ The conventional intruder-state avoidance technique (ISA) was used with a shift of $0.02 \mathrm{au}$ in these calculations. All MR calculations were performed with help of the Firefly program suite (version 8.1.0). To avoid the problem of selfconsistency of MR perturbation theory calculations, all products of the reaction(s) were considered in the calculation with the same active space as for the original molecule, but separated by about $20 \AA$. Albeit deficient in the absence of evaluation of ZPE and entropy contributions (which can notably change the final numbers), the method provides a unique opportunity to precisely analyze different contributions to the total wavefunction, especially in the case when the system cannot be described by a single determinant. This is precisely the case for any transition state considered herein, as well as a significant number of ground-state molecules.

Keywords: breaking into cores - cesium - fluorides . polyfluoride anions $\cdot$ quantum chemistry

How to cite: Angew. Chem. Int. Ed. 2015, 54, 8275-8278 Angew. Chem. 2015, 127, 8393-8396

[1] a) P. F. Walch, D. E. Ellis, J. Chem. Phys. 1976, 65, 2387-2392; b) V. A. Gubanov, A. Rosén, D. E. Ellis, J. Inorg. Nucl. Chem. 1979, 41, 975-986; c) K. Tatsumi, R. Hoffmann, Inorg. Chem. 1980, 19, 2656; d) P. Belanzoni, E. J. Baerends, E. Van Lenthe, Mol. Phys. 2005, 103, 775-787; e) "Theoretical Studies of the Electronic Structure of Compounds of the Actinide Elements": N. Kaltsoyannis, P. J. Hay, J. Li, J.-P. Bladeau, B. E. Bursten in The Chemistry of the Actinide and Transactinide Elements, Vol. 3 (Eds.: L. Morss, N. M. Edelstein, J. Fuger), Springer, Berlin, 1893-2011, 2006; f) R. G. Denning, J. Phys. Chem. A 2007, 111 , 4125; g) P. Pyykkö, Chem. Rev. 2012, 112, 371-384; h) M. D. Neidig, D. L. Clark, R. L. Martin, Coord. Chem. Rev. 2013, 257, 394.

[2] a) S. Riedel, M. Straka, M. Kaupp, Chem. Eur. J. 2005, 11, 2743, and references therein; b) X. Wang, L. Andrews, S. Riedel, M. Kaupp, Angew. Chem. Int. Ed. 2007, 46, 8371; Angew. Chem. 2007, 119, 8523 .

[3] In recent work, one of us found that pressure can stabilize high oxidation states of $\mathrm{Hg}$ in an extended material: J. Botana, $\mathrm{X}$ Wang, D. Yang, H. Lin, Y. Ma, M. Miao, unpublished results.

[4] W. Grochala, R. Hoffmann, J. Feng, N. W. Ashcroft, Angew. Chem. Int. Ed. 2007, 46, 3620; Angew. Chem. 2007, 119, 3694.

[5] M. Miao, Nat. Chem. 2013, 5, 846-852.

[6] K. O. Christe, J. Am. Chem. Soc. 1991, 113, 3351.

[7] The possible existence of cesium fluorides has been probed experimentally: a) H. Bode, Naturwissenschaften 1950, 37, 477; b) K. Moock, K. Seppelt, Angew. Chem. Int. Ed. Engl. 1989, 28 , 1676; Angew. Chem. 1989, 101, 1713; c) L. B. Asprey, J. L. Margrave, M. E. Silverthorne, J. Am. Chem. Soc. 1961, 83, 2955 d) C. Jehoulet, A. J. Bard, Angew. Chem. Int. Ed. Engl. 1991, 30, 836-838; Angew. Chem. 1991, 103, 882-884; the experimental verdict so far appears to be "not proven".

[8] $\mathrm{CsF}_{3}$ is known from matrix isolation studies. This fascinating molecule is a $\mathrm{Cs}^{+}$complex of the trifluoride ion: B. S. Ault, L. Andrews, J. Am. Chem. Soc. 1976, 98, 1591.

[9] T. Vent-Schmidt, F. Brosi, J. Metzger, T. Schlöder, X. Wang, L. Andrews, C. Müller, H. Beckers, S. Riedel, Angew. Chem. Int. Ed. 2015, 54, 8279-8283; Angew. Chem. 2015, 127, 8397-8401.
[10] $\mathrm{CsF}$ is a common ionic solid with a distance in the solid of $3.01 \AA$ : W. Pies, A. Weiss, a1, I.1.1 Simple fluorides and their solid solutions. SpringerMaterials-The Landolt-Börnstein Database (Eds.: K.-H. Hellwege, A. M. Hellwege), (http://www. springermaterials.com). DOI: 10.1007/10201462_2.

[11] For example, PBE0/QZVPP/ZORA gives $-34 \mathrm{kcal} \mathrm{mol}^{-1}$, whereas the combination of an MP2 approach with orbital optimization as well as with spin-component-scale techniques (OO-SCS-MP2/TZVP/ZORA) gives $-28 \mathrm{kcal} \mathrm{mol}^{-1}$. Details of these calculations are collected in the Supporting Information.

[12] K. P. Huber, G. Herzberg, Constants of Diatomic Molecules, 1979, Van Nostrand Reinhold.

[13] http://webbook.nist.gov/chemistry/.

[14] The previously computed structure of the most stable isomer of $\mathrm{CsF}_{3}$ (Figure 14 in H. Haller, S. Riedel, Z. Anorg. Allg. Chem. 2014, 640,1281) corresponds in our calculations to the transition state between two identical isomers of $\mathrm{CsF}_{3}$ (side-on isomer, central picture in our Figure S18). Our calculated barrier separating the isomers is low (ca. $1 \mathrm{kcal} \mathrm{mol}^{-1}$ ).

[15] a) S. Riedel, T. Köchner, X. Wang, L. Andrews, Inorg. Chem. 2010, 49, 7156; b) for experimental evidence for $\mathrm{F}_{5}{ }^{-}$see the discussion in Ref. [14]; c) A. Artau, K. E. Nizzi, B. T. Hill, L. S. Sunderlin, P. G. Wenthold, J. Am. Chem. Soc. 2000, 122, 10667.

[16] a) A. A. Tuinman, A. A. Gakh, R. J. Hinde, R. N. Compton, J. Am. Chem. Soc. 1999, 121, 8397-8398.

[17] Since trifluoride $\mathrm{F}_{3}{ }^{-}$is known experimentally, we also computed the energetics of fragmentation of $D_{5 h} \mathrm{CsF}_{5}$ to $\mathrm{Cs}^{+}+\mathrm{F}_{2}+\mathrm{F}_{3}$ $\left(\Delta E=+61 \mathrm{kcal} \mathrm{mol}^{-1}\right.$ at the PBE0 level).

[18] Q. Zhu, A. R. Oganov, Q. Zeng, Sci. Rep. 2015, 5, 785.

[19] A. Yu. Rogachev, R. Hoffmann, unpublished results.

[20] I. A. Krouse, C. Hao, C. E. Check, K. C. Lobring, L. S. Sunderlin, P. G. Wenthold, J. Am. Chem. Soc. 2007, 129, 846-852.

[21] N. Vasdev, M. D. Moran, H. M. Tuononen, R. Chirakal, R. J. Suontamo, A. D. Bain, G. J. Schrobilgeb, Inorg. Chem. 2010, 49, $8997-9004$.

[22] D. J. Grant, T.-H. Wang, D. A. Dixon, Inorg. Chem. 2010, 49, $261-270$.

[23] H. Rzepa, http://www.ch.imperial.ac.uk/rzepa/blog/?p=11681.

[24] a) C. F. Guerra, J. Snijders, G. te Velde, E. Baerends, Theor. Chem. Acc. 1998, 99, 391; b) G. te Velde, F. Bickelhaupt, E. Baerends, C. Guerra, S. Van Gisbergen, J. Snijders, T. Ziegler, J. Comput. Chem. 2001, 22, 931.

[25] Concerning the SO, it is tempting to note that at the limit of large $\mathrm{SO}$ (jj coupling), cesium in the oxidation state $\mathrm{V}$ would formally completely empty the semi-core $5 p_{3 / 2}$ subshell, and leave the $5 p_{1 / 2}$ subshell completely filled. A reviewer has suggested the following line of reasoning: Taking the SO from Desclaux's tables (At. Data Nucl. Data Tables 1973, 12,312) it is $42.55 \mathrm{kcalmol}^{-1}$. Multiplying by $4 / 3$, one gets for first-order SO $56.3 \mathrm{kcal} \mathrm{mol}^{-1}$. Actually, in a filled-shell molecule, only second-order SO effects should occur. Guessing an energy denominator of 1 au or $627.5 \mathrm{kcal} \mathrm{mol}^{-1}$, one gets $56.339^{2} / 627.5=5.058 \mathrm{kcal} \mathrm{mol}^{-1}$. This must still be multiplied by the $5 p$ character of these molecular orbitals, and corrections for $\mathrm{jj}$ vs. LS coupling. Against this orderof-magnitude estimate, the value of $1.33 \mathrm{kcal} \mathrm{mol}^{-1}$ is smallish, but not absurd. For this type of reasoning, see: P. Pyykkö, Chem. Rev. 1997, 97, 597.

[26] F. Neese, ORCA 2009, University of Bonn, Bonn, Germany.

[27] A. A. Granovsky, J. Chem. Phys. 2011, 134, 214113.

Received: January 15, 2015

Published online: June 3, 2015 